\title{
Diagnostic Fiberoptic Endoscopic
}

\section{Examination}

National Cancer Institute

\section{Source}

National Cancer Institute. Diagnostic Fiberoptic Endoscopic Examination. NCI Thesaurus.

Code C155920.

A diagnostic procedure in which a fiberoptic endoscope is used. 